\title{
Use of extracorporeal membrane oxygenation in pediatric thoracic organ transplantation
}

Paul M. Kirshbom, MD

Nancy D. Bridges, MD

Richard J. Myung, MD,

J. William Gaynor, MD

Bernard J. Clark, MD

Thomas L. Spray, MD
From the Departments of Cardiothoracic Surgery and Cardiology, Children's Hospital of Philadelphia, Philadelphia, Pa.

Supported in part by the Daniel M. Tabas Endowed Chair in Pediatric Cardiothoracic Surgery.

Received for publication April 4, 2001; revisions requested May 22, 2001; revisions received June 27, 2001; accepted for publication July 2, 2001

Address for reprints: J. William Gaynor, MD, Cardiothoracic Surgery, Rm 8527, Children's Hospital of Philadelphia, 34th St and Civic Center Blvd, Philadelphia, PA 19104 (E-mail: gaynor@email.chop.edu).

J Thorac Cardiovasc Surg 2002;123:130-6

Copyright (C) 2002 by The American Association for Thoracic Surgery

$0022-5223 / 2002 \$ 35.00+0 \quad \mathbf{1 2 / 1 / 1 1 8 5 0 4}$

doi:10.1067/mtc.2002.118504
Objective: Mechanical cardiorespiratory support is occasionally required before or after pediatric thoracic organ transplantation. Extracorporeal membrane oxygenation is the most commonly used mechanical support technique in children. The goal of this study was to examine the indications for initiation and outcomes after peritransplant use of extracorporeal membrane oxygenation.

Methods: A retrospective study was conducted of 65 patients who received peritransplant extracorporeal membrane oxygenation between November 1994 and June 2000. The pretransplant group included 45 patients (average age, 38 months) supported with extracorporeal membrane oxygenation and listed for transplantation (31 heart, 8 lung, and 6 heart-lung), and the post-transplant group included 20 patients (average age, 83 months) who required extracorporeal membrane oxygenation after thoracic organ transplantation (12 heart, 6 lung, and 2 heart-lung transplants). Hospital course and outcomes were evaluated.

Results: With regard to pretransplant extracorporeal membrane oxygenation, patients listed for heart transplants were more likely to survive to transplantation than were those listed for lung or heart-lung transplants (12/31 [39\%] vs 1/14 [7\%], $P=.03)$. There was no difference in long-term survival between heart transplant patients after extracorporeal membrane oxygenation and those without extracorporeal membrane oxygenation (12-month actuarial survival, $83 \%$ vs $73 \% ; P=.68$ ). Patients who survived for prolonged periods on extracorporeal membrane oxygenation ( $>250$ hours) typically received heart transplants (7/8 [88\%]). With regard to post-transplant extracorporeal membrane oxygenation, patients receiving lung or heart-lung transplants had better short-term outcomes than those receiving heart transplants (63\% survived to discharge vs $33 \%$ ). All 3 patients with early graft dysfunction receiving lung transplants survived to discharge.

Conclusions: Long-term outcomes among those undergoing heart transplantation after support with an extracorporeal membrane oxygenator are comparable with those of patients not receiving extracorporeal membrane oxygenation. Extracorporeal membrane oxygenation can be a useful post-transplant support device, particularly in patients undergoing lung transplants.

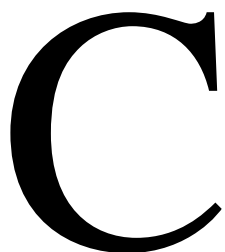

hildren listed for thoracic organ transplantation may require circulatory or pulmonary support either while waiting for organs to become available or at some point in the post-transplant period. Extracorporeal membrane oxygenation (ECMO) remains the most commonly used mechanical circulatory support system in children. Numerous case reports and series have described the use of ECMO as a bridge to transplantation or for support after thoracic organ transplantation ${ }^{1-6}$; however, the number of patients included in most of these reports has been small, 
TABLE 1. Patient characteristics

\begin{tabular}{|c|c|c|c|c|c|c|c|}
\hline Group & No. & $\begin{array}{l}\text { Age } \\
\text { (mo) }\end{array}$ & $\begin{array}{c}\text { Weight } \\
\text { (kg) }\end{array}$ & $\begin{array}{c}\text { Sex } \\
(\mathrm{M} / \mathrm{F})\end{array}$ & $\begin{array}{c}\text { Pre-ECMO } \\
\text { arrest }\end{array}$ & $\begin{array}{c}\text { ECMO } \\
\text { duration (h) }\end{array}$ & $\begin{array}{c}\text { Transplanted } \\
\text { (\%) }\end{array}$ \\
\hline \multicolumn{8}{|c|}{ Bridge to transplant } \\
\hline Heart & 31 & 38 & 11.2 & $14 / 17$ & $11(35 \%)$ & 224 & $12(39 \%)$ \\
\hline Lung & 8 & 23 & 7.9 & $4 / 4$ & $3(38 \%)$ & 588 & 0 \\
\hline Heart-lung & 6 & 14 & 6.7 & $3 / 3$ & $1(17 \%)$ & 278 & $1(17 \%)$ \\
\hline \multicolumn{8}{|c|}{ Post-transplant ECMO } \\
\hline Heart & 12 & 83 & 35.0 & $7 / 5$ & $5(42 \%)$ & 157 & NA \\
\hline Lung & 6 & 90 & 20.6 & $2 / 4$ & $1(17 \%)$ & 203 & NA \\
\hline Heart-lung & 2 & 215 & 29.5 & $0 / 2$ & $2(100 \%)$ & 52 & NA \\
\hline
\end{tabular}

Age, weight, and duration are presented as means.

$N A$, Not applicable.

and the majority of the patients undergoing lung transplantation have been adults.

The goal of this study was to retrospectively evaluate our experience with the use of ECMO in children immediately before or after thoracic organ transplantation at a single institution. For the purposes of this study, bridge to transplant was defined as the use of ECMO in any child who was listed for primary thoracic organ transplantation at some time during the period of ECMO support. Patients who were placed on ECMO immediately after transplantation were not included in the bridge-to-transplant group, even if they were relisted during that period.

\section{Patients and Methods}

The patient population was defined as those patients who were listed for transplantation at the Children's Hospital of Philadelphia between November 1994 and June 2000 who received ECMO support either while listed for primary transplant or at some point after transplantation. The patients were identified by cross-referencing the thoracic organ transplant database with the ECMO perfusion database. A total of 232 patients were listed for 276 organs during this time period, including 136 hearts, 95 lungs, and 45 heart-lung blocks. Of these patients, $126(54 \%)$ received 144 transplants (83 heart, 40 lung, and 21 heart-lung transplants). ECMO was used for support in 65 (28\%) of the 232 patients listed: 45 received support at some point in the primary pretransplant period, and 20 patients were placed on ECMO at some time after their initial transplant. These 65 patients comprise the study population. Three of the 65 patients were supported with a ventricular assist device (VAD) either before or after ECMO support and were included in the study. Two patients were supported with VADs without ECMO support at any time and were not included in the analysis.

Hospital and outpatient records were reviewed, and data were collected concerning hospital course and long-term survival. This study protocol was reviewed and approved by the Children's Hospital of Philadelphia Institutional Review Board.

\section{ECMO Techniques}

A standardized ECMO perfusion circuit was used with appropriately sized cannulas on the basis of patient size. Transthoracic cannulation (aorta and right atrium) was used in 21 patients (14 pretransplant postcardiotomy patients and 7 post-transplant patients). Cervical cannulation (typically right carotid artery and internal jugular vein) was used in the remaining 44 patients. Pump flows were regulated on the basis of systemic perfusion, oxygenation, and mixed venous oxygenation. Pharmacologic support was weaned as tolerated.

Routine echocardiography was used to evaluate ventricular function and cardiac anatomy and to rule out mediastinal clot or fluid collections in the postcardiotomy patients. Postcardiotomy patients frequently required reexploration in the intensive care unit to evacuate clots or to place a left atrial vent if left-sided distention was noted on echocardiography. Five patients who were cannulated with the cervical approach required transcatheter atrial balloon septostomy for left-sided decompression. All patients were maintained on prophylactic antibiotics to cover Gram-positive organisms unless culture-directed antibiotic coverage was indicated.

ECMO support was continued until one of the following end points was achieved: recovery of function sufficient to support stable hemodynamics; availability of a transplant organ; or development of complications rendering the patient a noncandidate for transplant or recovery. There was no preestablished time limit for ECMO support.

\section{Statistical Analysis}

Data are presented as means $\pm \mathrm{SD}$. Intergroup comparisons were carried out with unpaired $t$ tests. Comparison of survival between groups was carried out with Kaplan-Meier analysis with the CoxMantel test. Because of similarities between the lung and heartlung transplant patient populations, these 2 groups were combined to form a lung-containing transplant group for some statistical comparisons.

\section{Results}

\section{Patient Characteristics}

Summary data for all groups are presented in Table 1 . The post-transplant ECMO group was older and heavier than the bridge-to-transplant group as a whole, but there was not a significant difference in sex distribution among the groups. About one third of the patients had a cardiac arrest before initiation of ECMO, again with no difference between the bridge-to-transplant and post-transplant ECMO groups. 
TABLE 2. Diagnoses of pre-heart-transplant ECMO patients

\begin{tabular}{lc}
\hline Diagnosis & No. (\%) \\
\hline Postcardiotomy patients & $17(55 \%)$ \\
Hypoplastic left heart syndrome & 5 \\
Transposition of the great arteries & 4 \\
Other diagnoses & 8 \\
Noncardiotomy patients & $14(45 \%)$ \\
Cardiomyopathy & 7 \\
Myocarditis & 2 \\
Other diagnoses & 5 \\
\hline
\end{tabular}

For patients who were placed on ECMO in the pretransplant period, the likelihood of surviving to transplant was highly organ related. Patients listed for heart transplant survived to transplantation almost $40 \%$ of the time, whereas only 1 (7\%) patient listed for either lung or heart-lung transplant underwent transplantation. This occurred despite significantly longer duration of ECMO in the lung/heart-lung group ( $455 \pm 412$ hours vs $224 \pm 230$ hours for heart transplant patients, $P=.02$ ).

\section{Bridge to Transplant}

Heart transplant patients. Of the 31 patients who were placed on ECMO and listed for heart transplantation, 6 demonstrated sufficient recovery of function to be weaned from ECMO and survived to discharge from the hospital (bridged to recovery). The average duration of ECMO for these 6 patients was 4.4 days (range, 1.4-7.9 days). Of the remaining 25 patients, 1 was weaned from ECMO and received a transplant the following day, and 11 underwent transplantation while on ECMO, for a total of 12 patients successfully bridged to transplant. Thus, $18(58 \%)$ of the 31 patients had a positive outcome, which was defined as either survival to transplant (bridged to transplant) or weaning from ECMO with survival to discharge (bridged to recovery). The remaining 13 patients either had complications while on ECMO that rendered them noncandidates for transplant, at which time support was withdrawn $(n=6)$, or were weaned from ECMO and did not survive to either transplant or discharge $(n=7)$. Overall, $15(48 \%)$ of the 31 patients survived to discharge.

Surprisingly, the occurrence of a cardiac arrest before initiation of ECMO had no bearing on either the likelihood of receiving a transplant or the likelihood of a positive outcome. Of the patients who had a cardiac arrest, $45 \%(5 / 11)$ did not receive a transplant, as opposed to $35 \%(7 / 20)$ of those who did not have an arrest $(P=.58)$. Also, 64\% $(7 / 11)$ of those who had a cardiac arrest had a positive outcome, as opposed to 55\% (11/20) of those who did not $(P=.65)$.

The cause of cardiac disease, however, was a significant factor for both likelihood of transplant and positive out-
TABLE 3. Most common complications occurring on ECMO for pretransplant and post-transplant support (including all groups)

\begin{tabular}{lcc}
\hline Complication & Pretransplant, $\mathbf{n}(\%)$ & Post-transplant, $\mathbf{n}(\%)$ \\
\hline Sepsis (bacterial or fungal) & $7(16)$ & $1(5)$ \\
Hemorrhage & $6(13)$ & $5(25)$ \\
Code-related neurologic injury & $5(11)$ & $1(5)$ \\
Intracerebral hemorrhage & $4(9)$ & $1(5)$ \\
Multisystem failure & $3(7)$ & $1(5)$ \\
Renal failure & $2(4)$ & $2(10)$ \\
\hline
\end{tabular}

come. The group was divided into those patients with cardiac dysfunction after cardiotomy $(\mathrm{n}=17)$ and those with other diagnoses (noncardiotomy patients, $\mathrm{n}=14$ ). Patient diagnoses are shown in Table 2. Only 18\% (3/17) of the postcardiotomy patients survived to transplant, as opposed to $64 \%(9 / 14)$ of the noncardiotomy patients $(P<.01)$. Four postcardiotomy and 2 noncardiotomy patients were weaned from ECMO and survived to discharge, and therefore $41 \%$ (7/17) of the postcardiotomy patients had a positive outcome, as opposed to $79 \%(11 / 14)$ of the noncardiotomy patients $(P=.04)$. Four postcardiotomy patients had complications resulting in death on ECMO, and 6 died an average of 30 days (range, 7-53 days) after weaning from ECMO. The majority of the 10 postcardiotomy patients who died had a combination of sepsis and multisystem organ failure, with the exclusion of 1 code-related neurologic injury (allowed to die on day 1 of ECMO) and 1 patient with isolated renal failure leading to multiple complications (Table 3). Only 2 of the postcardiotomy ECMO patients underwent surgical procedures while on ECMO (excluding mediastinal explorations for hematoma removal). One patient underwent a patch aortoplasty to correct an ascending aortic stenosis, and a second patient had a residual atrial septal defect closed with concomitant pulmonary arterioplasty to correct a pulmonary artery stenosis. Both of these patients were weaned from ECMO and later died at 28 and 53 days after decannulation, respectively. Outcomes for postcardiotomy patients with a functional single ventricle was not different than those for the group as a whole. Of the 6 patients with single-ventricle physiology (5 after stage 1 correction and 1 after the Fontan procedure), 1 patient underwent transplantation and is a long-term survivor, 1 was weaned and is alive 1.5 years later, 2 died while on ECMO, and 2 died late after weaning from ECMO.

Three of the 14 noncardiotomy patients did not have a positive outcome. One of these patients had a glycogen storage disease that was not believed to be compatible with long-term survival and was allowed to die. One patient had multisystem organ failure while on ECMO and died. The last patient, who had a diagnosis of Ebstein malformation, 


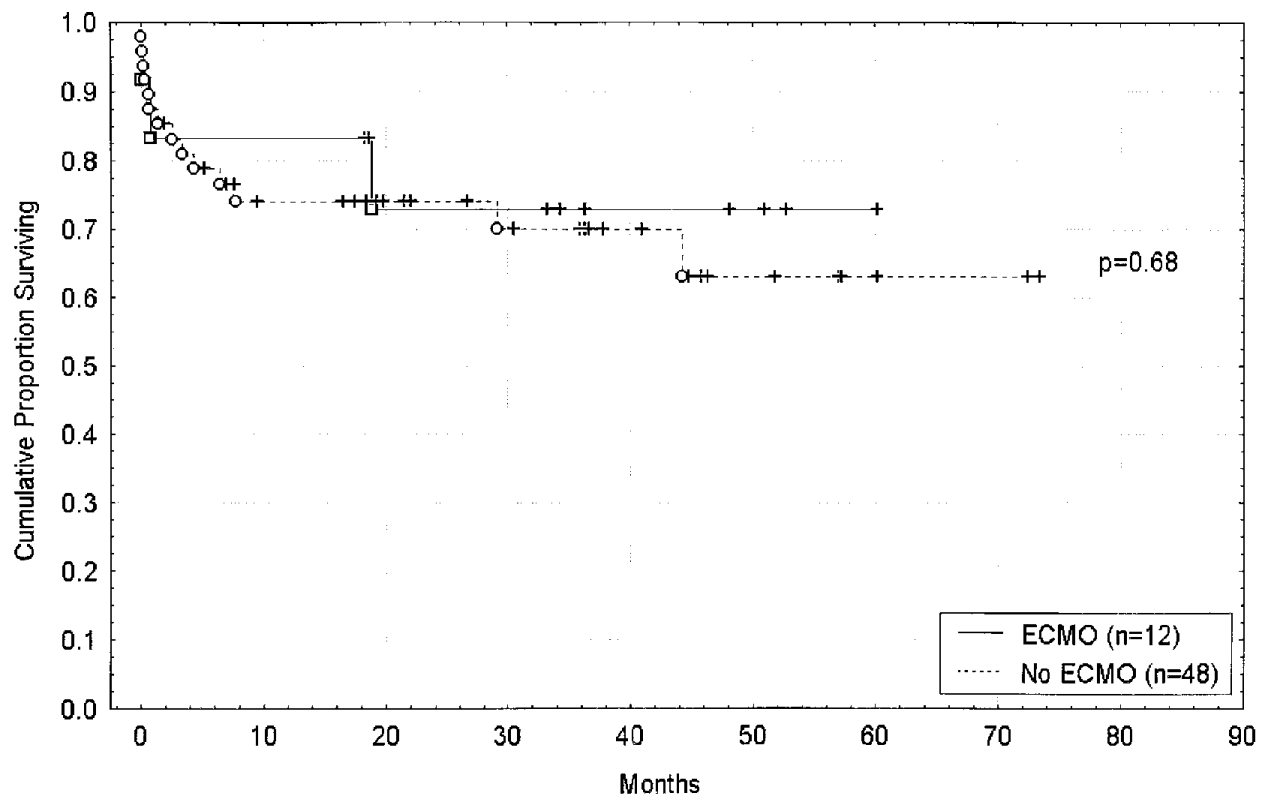

Figure 1. Survival after primary heart transplantation for pretransplant ECMO versus no ECMO.

died of respiratory failure complicated by pneumonia 58 days after weaning from ECMO.

The long-term outcome of patients who were successfully bridged to heart transplant $(n=12)$ was compared with that of those patients who underwent transplantation during this period without preoperative ECMO support $(n=48$, Figure 1). There was no significant difference in survival between these groups, with a 12-month actuarial survival of $83 \%$ for the ECMO patients versus $73 \%$ for the non-ECMO patients $(P=.68)$.

Lung and Heart-Lung Transplant Patients. As can be seen from the data presented in Table 1, patients listed for lung or heart-lung transplantation who required pretransplant ECMO were unlikely to survive to transplantation. Of the 14 patients in this group, only 1 patient underwent transplantation (a heart-lung patient), and he is alive at 13 months after transplantation. Of the remaining 13 patients, 1 child (listed for heart-lung transplant) was successfully weaned and discharged home. This child, who had a previously repaired atrioventricular canal defect, had cardiopulmonary dysfunction after left atrioventricular valve replacement and is alive 19 months after ECMO. The remaining 12 children, including all of those listed for lung transplantation, did not survive to transplant or discharge.

Given the small number of patients who received a transplant or survived to discharge, statistical comparisons are impossible. However, some interesting information can be gleaned. First, all of the patients with pre-ECMO cardiac arrests had documented pulmonary hypertension. Of the 10 patients in the lung/heart-lung transplant group with pul- monary hypertension, $4(40 \%)$ had a pre-ECMO arrest. These 4 patients had an average ECMO duration of 106 hours, and they were all removed from ECMO support because of arrest-related neurologic injuries. The 5 pulmonary hypertensive patients who did not have cardiac arrests and who did not receive transplants had an average ECMO duration of 482 hours. Interestingly, the only patient who received a transplant carried a diagnosis of pulmonary hypertension, was cannulated electively, and underwent transplantation 13 hours later. None of the 4 patients without pulmonary hypertension had a cardiac arrest, and they were maintained on ECMO for an average of 855 hours until they either recovered function $(n=1)$ or had complications and were deemed not to be transplant candidates $(n=3)$.

\section{Post-transplant ECMO.}

Twenty patients required ECMO support at some point after transplantation (12 heart, 6 lung, and 2 heart-lung transplants). Early outcomes and the frequency of retransplantation are shown in Table 4. Of the 12 heart-transplant patients placed on ECMO after transplantation, 9 required support in the early (<7 days) post-transplant period, and 3 required support later in their course. Five of the patients who required early support experienced immediate graft dysfunction or right ventricular failure during the initial transplant and were transitioned to ECMO from cardiopulmonary bypass. Three of the remaining 4 patients requiring early support had cardiac arrests within 24 hours of the initial transplant, and the last patient was placed on ECMO for progressive hemodynamic instability. Four of the 9 patients 
TABLE 4. Outcomes after post-transplant ECMO

\begin{tabular}{lcccc}
\hline Group & No. & Relisted & Retransplanted & $\begin{array}{c}\text { Survived to } \\
\text { discharge, } \\
\mathbf{n}(\%)\end{array}$ \\
\hline Heart & 12 & 6 & 2 & $4(33)$ \\
$\quad$ Early & 9 & 4 & 2 & $2(22)$ \\
Late & 3 & 2 & 0 & $2(66)$ \\
Lung/heart-lung & 8 & 6 & 4 & $5(63)$ \\
$\quad$ Early & 3 & 2 & 1 & $3(100)$ \\
Late & 5 & 4 & 3 & $2(40)$ \\
\hline
\end{tabular}

requiring ECMO early after heart transplantation were listed for retransplantation, with 2 of these patients receiving organs. One of the 2 patients having retransplantation was discharged from the hospital, and 1 other child was weaned from ECMO and also survived to discharge. Of the 3 patients who required support in the late post-transplant period, 2 had episodes of rejection leading to hemodynamic instability, and the third had a cardiac arrest 9 years after the initial transplant operation. Two of these patients recovered function after treatment for rejection, were successfully weaned from ECMO, and survived to discharge.

Of the 8 patients who were supported with ECMO after lung/heart-lung transplants, 3 were supported in the early post-transplant period and 5 in the late post-transplant period. Of the 3 patients requiring early support, 2 were listed for retransplant, with 1 patient receiving a retransplant. Graft function improved in the remaining 2 patients who were weaned from ECMO. All 3 patients survived to discharge. Three of the 5 patients supported in the late posttransplant period underwent retransplantation, with 1 of these patients surviving to discharge. One of the 2 patients who were did not undergo retransplantation recovered graft function, was weaned from ECMO, and survived to discharge. Overall survival to discharge was 3 of $3(100 \%)$ for early post-transplant and 2 of $5(40 \%)$ for late post-transplant support after lung and heart-lung transplants.

\section{Discussion}

The options available for mechanical cardiopulmonary support in pediatric patients are limited in most institutions because of the lack of VADs appropriate for a wide range of sizes. Also, the frequency of biventricular dysfunction and pulmonary hypertension in the pediatric population often makes the application of VADs difficult even if they are available. Placement of bilateral VADs in small children can be difficult and introduces a variety of potential complications. Because of these problems and the widespread use of ECMO for support of children with respiratory failure, ECMO remains the most commonly used method of mechanical circulatory support in children.
There are several advantages and disadvantages to ECMO that must be considered. The most obvious advantage of ECMO is the familiarity of most programs with this method of support. ECMO circuits can be prepared for use quickly, and patients can be cannulated and support initiated in the intensive care unit if necessary. VADs, on the other hand, require a thoracotomy or sternotomy and a trip to the operating room. In emergency situations ECMO support can be instituted in 15 to 20 minutes. Another advantage of ECMO is its versatility. Essentially all forms of cardiopulmonary failure and all cardiac anatomic diagnoses can be treated with ECMO, including biventricular failure, right-sided failure with pulmonary hypertension, and isolated left-sided failure. VADs, on the other hand, are ideally suited to isolated failure of 1 ventricle in a biventricular heart. Although dual ventricular support is possible and not uncommonly used in adults, space considerations make bi-VAD support problematic in small children. The most significant disadvantages of ECMO are the requirements for immobilization, intensive care monitoring, and anticoagulation. Unlike some patients receiving intracorporeal VAD support, children on ECMO cannot be extubated and mobilized.

For patients awaiting transplantation, the duration of ECMO support is generally determined by organ-allocation policies rather than patient-specific characteristics. We have previously reported our experience with long-term pretransplant ECMO support for a patient who required 1126 hours of support before a heart became available. ${ }^{7}$ Although this patient clearly represents the extreme, 4 of our patients have undergone transplantation after more than 500 hours of ECMO support, and of the 8 patients listed for heart transplants who remained on ECMO for more than 250 hours, 7 received transplants. These experiences indicate that there should be no arbitrary cutoff point for duration of support, so long as complications precluding transplantation have not developed.

Several reports have discussed the use of ECMO as a bridge to cardiac transplantation in children. The largest series in the literature was that of del Nido and colleagues, ${ }^{3}$ which included 14 children placed on ECMO and listed for heart transplantation between 1981 and 1993. Several interesting points can be gleaned from a comparison between their experience and ours. First, postcardiotomy patients had a worse outcome than noncardiotomy patients in both studies, with $25 \%(2 / 8)$ of the postcardiotomy patients surviving to discharge compared with $83 \%$ (5/6) of the noncardiotomy patients in the earlier study. Second, del Nido and colleagues received organs for $9(64 \%)$ of their 14 patients after an average ECMO duration of 102 hours, whereas in the current study, only $12(39 \%)$ of 31 patients listed for heart transplants received organs, despite an average ECMO duration of 359 hours. This difference reflects the increasing scarcity of donor organs in the interval between the 2 reports. However, despite increased organ 
scarcity and longer duration of ECMO support, there was no difference in short-term outcomes between the 2 studies: early survival for the group as a whole was $50 \%$ in the previous study compared with $48 \%$ in the current report.

The decision of when to list postcardiotomy patients for heart transplantation can be a difficult one because it is difficult to predict which patients might recover function. In this group the patients who were successfully weaned from ECMO had an average ECMO duration of 4.5 days, with a range of 1.4 to 7.9 days. Therefore, in general, those patients who will be successfully weaned from ECMO will do so within 8 days. Mehta and colleagues ${ }^{8}$ reported a similar ECMO duration of 3.4 days for their postcardiotomy patients who were successfully weaned from support. On the other side of the equation, the 3 postcardiotomy patients who were bridged to transplant received organs after 3, 8, and 10 days on the waiting list, respectively. The decision of when to list postcardiotomy patients for transplantation must be individualized on the basis of the management team's assessment of likelihood of recovery. Patients who are believed to be unlikely to recover cardiac function on the basis of anatomy and intraoperative evaluation are generally listed earlier than those who are believed to be more likely to recover function. In most circumstances patients are listed as soon as transplant candidacy is confirmed so as to maximize the patient's chance of receiving an organ before complications develop.

Although it may seem counterintuitive that a pre-ECMO cardiac arrest does not significantly affect outcome for patients listed for heart transplantation, previous studies have reported survival in the $33 \%$ to $53 \%$ range for these patients, ${ }^{3,8}$ which is consistent with the current report. For those patients who are placed on ECMO after a cardiac arrest ( $\mathrm{n}=15$ : 11 heart, 3 lung, and 1 heart-lung) and who were not previously listed for transplantation (10/15), the timing of listing is variable and depends on exclusion of significant neurologic or other end-organ injury after the arrest. On average, these patients were listed for transplantation 1.4 days after the arrest, with a range of 0 to 8 days. At our institution, donor-organ size and distance-acceptance criteria are not altered for this group of patients, but suboptimal organs are occasionally considered.

Perhaps the most important finding of this study is that the long-term outcome for patients who receive heart transplants after ECMO bridge to transplant is not discernibly different from that of patients who undergo transplantation without pretransplant ECMO (Figure 1). This result is markedly different from the findings of Dellgren and colleagues, ${ }^{9}$ who reported significantly worse outcomes for post-ECMO transplant patients (2-year survival of approximately $48 \%$ vs $88 \%$ for non-ECMO patients, $P<.01)$. Given the results of the current study, aggregate outcomes might be further improved if organallocation protocols were modified so as to minimize waiting times for patients on ECMO or other mechanical support.
Unfortunately, the outcome for patients who required ECMO support while waiting for lung or heart-lung transplants was not as good as that of the heart-transplant patients. Only 1 patient of the pretransplant ECMO group received a heart-lung transplant, and none of the 8 patients listed for lungs survived to transplant. Equally disappointing was the low rate of successful weaning from ECMO, with only 1 patient surviving to discharge after discontinuation of support. Unlike patients with viral myocarditis or postcardiotomy myocardial dysfunction, whose cardiac function occasionally improves if given time with mechanical support, most children listed for lung transplants did not improve. The majority of patients listed for lung or heart-lung transplantation who required ECMO had pulmonary hypertension, with or without underlying cardiac disease. The outcome for patients with pulmonary hypertension who had a cardiac arrest while waiting for transplantation was particularly poor; all of these patients had neurologic injuries that rendered them noncandidates for transplant. Although we realize that the results are dismal for this group of patients, ECMO support is still selectively applied with the realization and understanding that support will be withdrawn early if neurologic or other end-organ injury precluding transplant is identified.

The literature concerning post-transplant support with ECMO is essentially the reverse of the pretransplant ECMO literature in that there are more reports of support after lung transplantation than after heart transplantation. In the current study survival after post-lung-transplant ECMO was $63 \%$ compared with $33 \%$ for the heart-transplant patients. The time course of support requirements and outcomes are interesting in that the majority of post-heart transplant patients required support early after transplantation $(9 / 12)$, with relatively poor outcomes in this group (2/9 survived to discharge). Only 3 patients required support in the late period after heart transplantation, with 2 of the 3 surviving to discharge. The lung/heart-lung transplant patients, on the other hand, were more evenly divided between the early and late groups (3 vs 5), and the early group had better early outcomes (100\% survival to discharge vs $40 \%$ ).

The difference in the outcome of patients requiring ECMO early after heart transplantation compared with lung/heart-lung transplantation is interesting. There have been several reports in the literature describing ECMO support after lung transplantation, primarily in adult patients. ${ }^{1,2,6}$ In these reports and the current study, patients with early or immediate reperfusion injury or graft dysfunction typically improved after 1 to 5 days of ECMO support. Patients who present late after transplant, however, do not recover function as often, and therefore ECMO support is in effect an attempt to bridge to retransplant. Lung-containing grafts injured by infectious processes, adenovirus in particular, often do not recover, and recipient survival requires retransplantation. ${ }^{10,11}$ 
In conclusion, ECMO can be an effective therapeutic tool for children who require or have received thoracic organ transplants. Patients who receive heart transplants after ECMO support can be expected to have long-term outcomes comparable with those of patients without a history of mechanical support, even if a cardiac arrest occurs before initiation of ECMO. Given the excellent outcomes that can be achieved for these patients if they can undergo transplantation before complications develop, consideration should be given to a change in organ-allocation policies so as to operate on these children as quickly as possible. In contrast to the heart-transplant group, ECMO patients listed for primary lung or heart-lung transplantation rarely survive to either transplant or discharge; this outcome might also be improved, although probably to a lesser degree, by a change in organallocation policies. Finally, ECMO can be a very effective method of post-transplant support for children, particularly lung-transplant patients with early graft dysfunction.

\section{References}

1. Ball JW, Noon GP, Short HD, Scheinin SA. Extracorporeal membrane oxygenation in lung transplantation: a case report. J Heart Lung Transplant. 1997;16:468-71.

2. Nguyen DQ, Kulick DM, Bolman RM, Dunitz JM, Hertz MI, Park SJ.
Temporary ECMO support following lung and heart-lung transplantation. J Heart Lung Transplant. 2000;19:313-6.

3. del Nido PJ, Armitage JM, Fricker FJ, Shaver M, Cipriani L, Dayal G, et al. Extracorporeal membrane oxygenation support as a bridge to pediatric heart transplantation. Circulation. 1994;90:II66-9.

4. Hopper AO, Pageau J, Job L, Heart J, Deming DD, Peverini R. Extracorporeal membrane oxygenation for perioperative support in neonatal and pediatric cardiac transplantation. Artif Organs. 1999;23: 1006-9.

5. Ko W-J, Chen Y-S, Chou N-K, Wang S-S, Chu S-H. Extracorporeal membrane oxygenation in the perioperative period of heart transplantation. J Formos Med Assoc. 1997;96:83-90.

6. Meyers BF, Thoralf M Sundt I, Henry S, Trulock EP, Guthrie T, Cooper JD, et al. Selective use of extracorporeal membrane oxygenation is warranted after lung transplantation. J Thorac Cardiovasc Surg. 2000;120:20-8.

7. DiRusso GB, Clark BJ, Bridges ND, Godinez RI, Paridon SM, Spray TL, et al. Prolonged extracorporeal membrane oxygenation as a bridge to cardiac transplantation. Ann Thorac Surg. 2000;69:925-7.

8. Mehta U, Laks H, Sadeghi A, Marelli D, Odim J, Alejos J, et al. Extracorporeal membrane oxygenation for cardiac support in pediatric patients. Am Surg. 2000;66:879-86.

9. Dellgren G, Koirala B, Sakopoulus A, Botta A, Joseph J, Benson L et al. Pediatric heart transplantation: improving results in high-risk patients. J Thorac Cardiovasc Surg. 2001;121:782-91.

10. Bridges ND, Spray TL, Collins MH, Bowles NE, Towbin JA. Adenovirus infection in the lung results in graft failure after lung transplantation. J Thorac Cardiovasc Surg. 1998;116:617-23.

11. Hoffman TM, Spray TL, Gaynor JW, CLark BJ, Bridges ND. Survival after acute graft failure in pediatric thoracic organ transplant recipients. Pediatr Transplant. 2000;4:112-7. 